\title{
SISTEMA INTEGRADO DE GESTÃO DE ATIVIDADES ACADÊMICAS SIGAA - MÓDULO BIBLIOTECA: UMA OPORTUNIDADE DE RETOMAR A CREDIBILIDADE DA COMUNIDADE ACADÊMICA COM A EFETIVAÇÃO DA GESTÃO DO SISTEMA DE BIBLIOTECAS DA UNIVERSIDADE FEDERAL DA PARAÍBA
}

\author{
INTEGRATED MANAGEMENT SYSTEM OF ACADEMIC ACTIVITIES SIGAA - MODULE \\ LIBRARY: AN OPPORTUNITY TO REGAIN THE CREDIBILITY OF THE ACADEMIC \\ COMMUNITY WITH A EFFECTIVE MANAGEMENT OF THE LIBRARY SYSTEM OF THE \\ FEDERAL UNIVERSITY OF PARAIBA
}

Maria das Graças Vieira

Fábio Firmino Machado ${ }^{2}$

\begin{abstract}
Resumo: Com a efetivação na Universidade Federal da Paraíba através de termo de cooperação técnica com a Universidade Federal do Rio Grande do Norte - UFRN, do Sistema Integrado de Gestão de Atividades Acadêmicas - SIGAA, em seus diversos módulos, com a implantação de sistemas informatizados de gestão de informações acadêmicas, administrativas e de recursos humanos como parte do processo de inovação tecnológica da UFPB, sendo abordado neste trabalho especificamente o módulo Biblioteca e percebendo a oportunidade de rever alguns paradigmas institucionais, o presente trabalho faz uma descrição do atual sistema informacional da Biblioteca Central da UFPB (coordenadora do sistema de bibliotecas - SISTEMOTECA), o Ortodocs e a transição para o novo sistema adquirido, com ênfase na integração de informações que para o autor pode dar visibilidade e real funcionalidade ao sistema de bibliotecas. Além de fornecer mais subsídios às tomadas de decisão, com mais propriedade e objetividade, surge como ferramenta de reaproximação com a "academia", ou seja, com os integrantes diretos e indiretos da comunidade universitária.
\end{abstract}

Palavras chave: Sistema de Informação. Inovação Tecnológica. Sistema de Bibliotecas

\begin{abstract}
With the effectuation of the Federal University of Paraíba by the technical cooperation term with Federal University of Rio Grande do Norte - UFRN, the Integrated Management System of Academic Activities SIGAA, on its various modules, with the implementation of academic, administrative, human resources management information systems as part of the technological innovation process of UFPB, being specifically approached in this article the Library module and realizing the opportunity to review some institutional paradigms, this research gives a description of the current information system of the Central Library of UFPB (coordinator of the library system - SISTEMOTECA), the Ortodocs and the transition to a new acquired system, with emphasis on information integration which according to the author can provide visibility and real functionality to the library system. In addition to providing more support to make decisions with more propriety and objectivity, it appears as a tool for rapprochement with the "academy", in other words, with direct and indirect members of the university community.
\end{abstract}

Keywords: Information System. Technological Innovation. Libraries System

\footnotetext{
${ }^{1}$ Doutora em Educação e Professora da Universidade Federal de Pernambuco (UFPE). E-mail: gracinhavieira@yahoo.com.br

${ }^{2}$ Mestrando em Gestão em Organizações (MPGOA-UFPB). E-mail: vicediretoria@ biblioteca.ufpb.br Enviado em: 16/07/2012 - Aceito em: 01/04/2013.

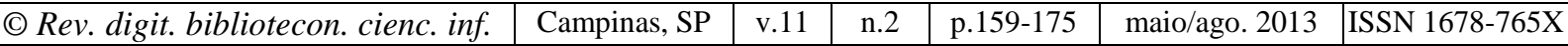




\section{INTRODUÇÃO}

O presente trabalho tem como principais premissas fazer uma descrição do atual sistema de informação utilizado pelo Sistema de Bibliotecas - SISTEMOTECA, o ORTODOCS coordenado pela Biblioteca Central - BC com suas principais características, limitações e dificuldades e em contraponto fazer uma analogia com a proposta de implantação já em andamento do novo Sistema Integrado de Gestão de Atividades Acadêmicas - SIGAA, com seus diversos módulos adquirido em 2010, através do Termo de Cooperação no 01/2010 da Universidade Federal da Paraíba - UFPB com a Universidade Federal do Rio Grande do Norte - UFRN. O módulo será implantado em todo o Sistema de Bibliotecas, porém o foco nesse estudo será especificamente nas ações necessárias a execução das atividades de implantação na Biblioteca Setorial - BS do Centro de Ciências da Saúde - CCS, tomada como piloto para validação do SIGAA.

$\mathrm{O}$ artigo enfoca a possibilidade de mudança paradigmática na gestão institucional, reflexo da integração das informações geradas que serão utilizadas como sustentáculo das decisões a serem tomadas doravante e como forma de aproximação dos segmentos formadores da academia, nesse ponto particulariza o Módulo Biblioteca como sendo a oportunidade de gerir efetivamente o SISTEMOTECA com a finalidade de reaver a credibilidade da comunidade acadêmica.

Este trabalho foi desenvolvido pelo autor na Biblioteca Central da UFPB em conjunto com os Analistas de Sistemas do NTI e com a participação dos Bibliotecários responsáveis pela setorial do CCS, o que possibilita comentar com propriedade a transição do atual para o novo sistema, tendo em vista estar vivenciando esse momento da perspectiva do gestor.

Durante o processo de implantação do módulo na BS do CCS, alguns fatos que impactaram diretamente o resultado desse relato precisam ser expostos: a negociação com o Núcleo de Tecnologia da Informação - NTI, com a finalidade de abreviar o período estabelecido em alguns meses, tendo em vista as peculiaridades das bibliotecas envolvidas, esse período foi antecipado em seis meses; em contraponto o longo intervalo de greve nas Universidades Federais prejudicou sensivelmente a evolução do processo. 
A implantação efetiva é condição prioritária para observação do que se propõe, a avaliação do SIGAA módulo Biblioteca como uma oportunidade de mudança paradigmática na gestão do SISTEMOTECA, para utilizá-lo como ferramenta de integração entre os segmentos acadêmicos, condição que ainda não foi alcançada e nesse contexto só será possível a avaliação quando o módulo estiver no processo de produção efetiva na BS do CCS, com todos os desvios mensurados e solucionados, com um lapso temporal para observação e validação do novo Sistema, que será abordado em novos estudos a serem desenvolvidos a partir desse levantamento.

Foram utilizados no ensaio, além da observação assistemática, a pesquisa documental e bibliográfica. Marconi e Lakatos (2002) definem a técnica da observação como um tipo de coleta de dados que faz uso dos sentidos para obtenção de determinados aspectos da realidade:

A observação ajuda o pesquisador a identificar e a obter provas a respeito de objetivos sobre os quais os indivíduos não têm consciência, mas que orientam seu comportamento. Desempenha papel importante nos processos observacionais, no contexto da descoberta, e obriga o investigador a um contato mais direto com a realidade. É o ponto de partida da investigação social. (MARCONI; LAKATOS, 2002, p.88)

A observação assistemática, também denominada espontânea, informal, simples, ocasional e acidental,

(...) consiste em recolher e registrar os fatos da realidade sem que o pesquisador utilize meios técnicos especiais ou precise fazer perguntas diretas. É mais empregada em estudos exploratórios e não tem planejamento e controle previamente elaborados. (MARCONI; LAKATOS, 2002, p.89)

Por sua vez, na pesquisa documental a fonte de coleta de dados está restrita a documentos, sejam escritos ou não, constituindo o que Marconi e Lakatos denominam "fontes primárias", as quais "podem ser recolhidas no momento em que o fato ou fenômeno ocorre, ou depois" (MARCONI; LAKATOS, 2002, p.62). No caso do presente estudo, as principais fontes de documentos foram, a internet e diversos materiais bibliográficos, especialmente artigos de periódicos científicos. 


\section{FUNDAMENTAÇÃO TEÓRICA}

Nos dias atuais torna-se cada vez mais evidente a necessidade de adaptação das instituições as inovações tecnológicas associadas à gestão da informação. Estamos inseridos na chamada Sociedade da Informação e do conhecimento que de acordo com Webster, é representada por uma sociedade na qual a informação é utilizada intensamente como elemento da vida econômica, social, cultural e política, dependendo de um suporte tecnológico para se propagar, demonstrando que esse processo se tornou um fenômeno social, instaurado dentro da sociedade.

Segundo Dias et al (2004, p.2) a informação é matéria prima para que os indivíduos participem das mudanças na realidade social, organizacional e na sua própria realidade. A sociedade que tem acesso à informação pode tanto dominar setores como desenvolvê-los (ADRIANI; ZOMER apud PESTANA et al 2003, p. 77).

Conforme Japiassú define (1996, p. 51), o conhecimento é uma apropriação intelectual de determinado campo, tendo em vista dominá-lo e utilizá-lo. Para Silva (2004, p. 144) uma informação é convertida em conhecimento quando um indivíduo consegue ligá-la a outras informações, avaliando-a e entendendo seu significado no interior de um contexto específico. Cabe ressaltar que o conhecimento não é estático, modificando-se mediante a interação com o ambiente, sendo esse processo denominado aprendizado (MORESI, 2000, p. 19).

Não se pode dissertar sobre Sociedade da Informação sem a Tecnologia da Informação. Nos pilares da chamada Sociedade da Informação, está a tecnologia (DAVENPORT, 1998 apud FERREIRA, 2003, 45). Segundo Pereira (1999) apud Ramos (2003, p. 15), a união das palavras "tecnologia e informação" começou a ser empregada na década de 80, para explicar a convergência de diversas correntes de desenvolvimento tecnológico, principalmente àquelas associadas à microeletrônica, às telecomunicações e à informática.

A informação como matéria prima na Sociedade da Informação tem mecanismos tecnológicos de componentes inter-relacionados, que coleciona, recupera, processa e distribui informação, a esse conjunto de componentes denominamos sistemas de informação. Sistema de Informação (em inglês, Information System) é a expressão utilizada para descrever um sistema automatizado (que pode ser denominado como Sistema de Informação Computadorizado), ou mesmo manual, que abrange pessoas, máquinas, e/ou métodos \begin{tabular}{l|l|l|l|l|l|l} 
(C) Rev. digit. bibliotecon. cienc. inf. & Campinas, SP & v.11 & n.2 & p.159-175 & maio/ago. 2013 & ISSN 1678-765X \\
\hline
\end{tabular} 
organizados para coletar, processar, transmitir e disseminar dados que representam informação para o usuário.

Uma das principais funções dos sistemas de informação é a de filtrar a informação para gerar conhecimento. Por isso, conforme Ferreira (2003, p.36), cada vez mais, grandes corporações vêm realizando investimentos vultosos em sistemas de informação, objetivando interagir de forma mais rápida e dinâmica em diversas áreas estratégicas. Surgem então os ERPs Sistemas de Integrados de informação que segundo Nunes (2007)

Os ERP, sigla inglesa de Enterprise Resource Planning, são sistemas integrados de informação de tipo back office. Os ERP integram os dados e processos de vários ou mesmo da totalidade dos departamentos da organização num único sistema: esta integração pode ser efectuada ao nível departamental ou funcional (sistemas finanças, marketing, comercial, pessoal, produção, etc.)

Outro conceito importante no ambiente de gestão organizacional são os Sistemas de Informações Gerenciais (SIGs) que compilam em um único banco de dados as informações geradas pelos vários sistemas integrados que servem como ferramenta nas tomadas de decisões.

Sistema de Informações Gerenciais (SIG) é o processo de transformação de dados em informações que são utilizadas na estrutura decisória da empresa, proporcionando, ainda, a sustentação administrativa para otimizar os resultados operados. (OLIVEIRA, 2005, p. 40)

A constante evolução tecnológica e a explosão de informações organizadas em sistemas de gestão da informação para geração de conhecimento consolidam as Organizações Aprendentes. David Garvin, autor de Building a learning organization, define as organizações que aprendem como aquelas "capacitadas a criar, a adquirir e a transferir conhecimentos e, ainda, a modificar seus comportamentos para refletir esses novos conhecimentos e insights". Considerando as universidades como organizações aprendentes e portanto em constante desenvolvimento institucional é relevante registrar que para Finger (1997) os processos de gestão universitária deveriam ser inovadores e melhorar a integração entre alunos, docentes, técnicos e em geral a comunidade universitária interna e externa. 


\title{
BIBLIOTECA CENTRAL E SISTEMA DE BIBLIOTECAS
}

A Biblioteca Central da UFPB é uma biblioteca universitária e como tal está inserida num contexto maior que são as universidades tendo grande relevância nos processos de pesquisa e inovação tecnológica, pois além do armazenamento do conhecimento científico produzido, fornece subsídios informacionais que fomentam o processo de pesquisa.

\begin{abstract}
As bibliotecas universitárias são organizações complexas, com múltiplas funções e uma série de procedimentos, produtos e serviços que foram desenvolvidos ao longo de décadas. No entanto, o seu propósito fundamental permaneceu o mesmo, isto é: proporcionar acesso ao conhecimento. Esse acesso ao conhecimento é que irá permitir que o estudante, o professor e o pesquisador possam realizar suas aprendizagens ao longo da vida (CUNHA, 2010).
\end{abstract}

A criação da Biblioteca Central - BC teve início em 1961 no Regimento da UFPB, contudo, só a partir de 11 de agosto de 1967 que surgiram os primeiros passos para sua criação efetiva.

Na época, a UFPB deu um passo decisivo para a implantação da Biblioteca Central Universitária, estabelecendo como obras prioritárias a construção do prédio, desde a primeira etapa de edificação do campus de João Pessoa.

A primeira proposta de Estruturação da Biblioteca Central foi elaborada pelo renomado Professor universitário e Bibliotecário Edson Nery da Fonseca, o projeto foi intitulado como "Teoria da Biblioteca Central".

A construção foi iniciada, mas não foi concluída. Foi instalada provisoriamente numa pequena sala do Instituto de Matemática, passando para a Biblioteca da Escola de Engenharia; posteriormente foi transferida para o prédio da antiga faculdade de educação e por fim para um edifício anexo ao da reitoria.

No final de 1976 teve início todo o processo de estruturação e implantação da Biblioteca Central, a partir da junção do acervo das treze Bibliotecas Setoriais.

Partindo então para a contratação de Bibliotecários, atualização do acervo de livros e periódicos, elaboração e aprovação do regulamento do Sistema de Bibliotecas, criação de 
novos serviços, automação dos técnicos, entre outros, culminando com a construção do prédio definitivo da Biblioteca Central com uma área construída de $8.500 \mathrm{~m}^{2}$.

Em 1980 o regulamento do Sistema de Bibliotecas foi aprovado pelo Conselho Universitário (CONSUNI), através da resolução 201/1980.

Em 2009 foi elaborado e aprovado o novo regulamento do sistema de bibliotecas pelo Conselho Universitário (CONSUNI), através da resolução 31/2009, que define Sistema de Bibliotecas como um conjunto de Bibliotecas integradas sob os aspectos funcional e operacional, tendo por objetivo a unidade e harmonia das atividades educacionais, científicas tecnológicas e culturais da UFPB, voltadas para a coleta, tratamento, armazenagem, recuperação e disseminação de informações, para o apoio aos programas de ensino, pesquisa e extensão.

A Biblioteca Central é formada pela Diretoria, Vice-Diretoria, Secretaria Administrativa, Setor de Contabilidade e por 3 (três) Divisões, que se subdividem em 11 (onze) Seções.

O sistema de bibliotecas da UFPB se apresenta hoje com a seguinte formação: 1 Biblioteca Central (coordenadora do sistema) e 17 Bibliotecas setoriais localizadas nos 4 campi. O SISTEMOTECA deveria funcionar de forma integrada e harmônica onde as informações seriam geradas e disseminadas uniformemente, deveria, porém a realidade do sistema não permite validar esse desejo, inúmeras são as causas dessa realidade: comunicação interna e externa deficitária, prioridades diversas dependo de cada centro, isolamento da Biblioteca Central nas tomadas de decisão que influenciam diretamente no processo de ensino, pesquisa e extensão, dentre outros de caráter estrutural e administrativos.

\section{ORTODOCS PARA SIGAA, UMA MUDANÇA NECESSÁRIA}

O SISTEMOTECA está passando por um processo de transição com a mudança de Sistema de Gerenciamento de Bibliotecas que Dutra e Ohira (2004) definem como:

[...] sistemas de bases de dados com uma finalidade especifica, projetados para controlar as atividades essenciais de uma biblioteca. Geralmente funcionam em computadores de grande porte, permitindo uma padronização, 
integração, compatibilidade e intercâmbio de um grande volume de informações [...].

Esse processo de mudança envolve a implantação do SIGAA módulo Biblioteca e a retirada definitiva do OrtoDocs, sistema de gerenciamento ainda em funcionamento, que é de propriedade da Empresa Potiron Informática.

A Potiron Informática, com sede na cidade de São Paulo e laboratório tecnológico em Campinas-SP é a empresa responsável pela criação e desenvolvimento do software para automação de bibliotecas OrtoDocs, que é um sistema para informatização de bibliotecas individuais ou interligadas em redes, com todas as suas atividades integradas: catalogação segundo o padrão AACR2 para todos os tipos de materiais; controle patrimonial, de circulação e de periódicos, pesquisa bibliográfica, aquisição de materiais, importação e exportação de dados e geração de relatórios, utilizando o software ReportSmith, tornando possível as saídas de dados impressas de acordo com as peculiaridades e necessidades de cada biblioteca. A biblioteca Central é detentora da licença de uso do software OrtoDocs, de acordo com o contrato de $n^{\circ}$ 01/2007 firmado entre a Universidade Federal da Paraíba e a Potiron Informática, que viabiliza o tratamento técnico das obras adquiridas, bem como sua disponibilização para uso da comunidade acadêmica prioritariamente, através de empréstimos, consultas e manuseio de material informacional de propriedade da UFPB.

Por conta da fragmentação dos sistemas informacionais da Universidade Federal do Rio Grande do Norte, que refletiam a falta de conexão e fluxo informacional inter-sistemas resultado da liberdade de escolhas dos diversos segmentos da instituição na aquisição de softwares que atendessem as demandas individuais dos diversos segmentos, surge a necessidade de unificação e integração dos sistemas numa única base de dados para dar maior autenticidade às informações geradas como suporte às tomadas de decisão. Foi criada em 2000 a Superintendência de Informática da UFRN com essa missão. Oliveira (2008) define os sistemas de informações gerenciais como processos utilizados para transformar dados em informações que auxiliem no processo decisório da empresa.

Um Sistema de Informações Gerencial (SIG) abrange uma coleção organizada de pessoas, procedimentos, software, banco de dados e dispositivos que fornecem informação rotineira aos gerentes e aos tomadores de decisão. O foco de um SIG é, principalmente, a eficiência operacional. Marketing, produção, finanças e outras áreas funcionais recebem suporte dos 
sistemas de informação gerencial e estão ligados através de um banco de dados comum (STAIR e REYNOLDS, 2002, p. 18).

Dentro desse contexto a Superintendência de informática da UFRN cria então os seguintes sistemas: SIGAA (acadêmico), SIPAC (patrimonial) e SIGRH (recursos humanos) dentre os quais o artigo enfatiza o SIGAA e mais particularmente o módulo Biblioteca.

A UFPB através de suas Pró-Reitorias e do Núcleo de Tecnologia da Informação após varias visitas técnicas com o objetivo de verificar a qualidade do sistema no desempenho das funções gerenciais a que se destina, formaliza em 29 de outubro de 2011, Termo de Cooperação com a UFRN, que tem como objeto promover a implantação de um novo sistema integrado de gestão acadêmica, administrativa e de recursos humanos na UFPB, bem como a gerência de seus dados, através de sistemas desenvolvidos pela UFRN e mantidos, após implantação, pela UFPB, mediante assessoria técnica prestada pela UFRN.

Contemplam também a capacitação e transferência de tecnologia da UFRN para a UFPB e as integrações da ferramenta a sistemas do governo federal como o SIAFI, SIAPE, SIASG e outros de interesse mútuo entre os partícipes.

Esse sistema tem como ponto fundamental a integração das informações acadêmicas geradas por professores, servidores e alunos com vínculo na instituição, isso possibilita ao usuário do sistema diversas opções de consultas e verificações em tempo real, se utilizando de login e senha previamente criados. As informações disponibilizadas pelo sistema vão desde a vida acadêmica do aluno, produção intelectual dos professores, até a mais recente mutação de acervo da biblioteca efetuada pela instituição.

O ORTODOCS que é o sistema de gestão de informação oficial da Biblioteca Central da UFPB foi adquirido em meados dos anos 2000, com a finalidade prioritária de automatizar todas as operações, processos e atividades rotineiras da unidade e à época foi uma decisão acertada e ousada da gestão da BC. Houve uma breve interrupção legal por conta da impossibilidade de se prorrogar o contrato por mais de cinco anos, porém por conta da exclusividade do produto oferecido pela Potiron, foi possível a elaboração de um novo contrato em março de 2007 com o n $^{\circ}$ 01/2007 e assim o sistema pode ser mantido.

Basicamente a estrutura do sistema consiste em dois módulos: circulação e processos técnicos. A implantação foi escalonada, primeiro foi implementado os processos técnicos e 
por fim a circulação, ambos demandaram esforços conjuntos da BC e do Núcleo de Tecnologia da Informação - NTI para que o desafio fosse exitoso. Em termos práticos o processo técnico é o responsável direto pelo tratamento da obra: classificação, catalogação e inserção na base de dados. A circulação uma vez estando disponibilizado no acervo ao usuário via sistema, se responsabiliza pelo fluxo de cadastro dos usuários e empréstimo das obras. Vale ressaltar que a implantação do sistema provocou a racionalização dos processos, e com isso as informações puderam ser geridas e disponibilizadas com maior eficiência e eficácia.

O módulo biblioteca do SIGAA compreende três processos fundamentais: aquisição, processamento técnico e circulação, indispensáveis para o correto registro das obras adquiridas pela instituição, a garantia da funcionalidade desses processos é premissa básica para o sucesso da implementação desse módulo. Em demonstração previa da operacionalidade do sistema promovida pela equipe de Tecnologia da Informação da UFRN foi possível detectar a existência de diversas opções adicionais e opcionais que agregam valor ao contexto.

Quando se analisa isoladamente os sistemas, o utilizado e o novo em fase de implantação verificam-se algumas similaridades nas funcionalidades e disposição das informações na sua interface, como por exemplo: formato web e catálogo online, porém, se diferenciam e distanciam quando executamos as mesmas tarefas nos dois ambientes.

Algumas diferenças entre os sistemas podem ser percebidas em pontos estratégicos e consideradas relevantes do ponto de vista gerencial, que são os seguintes:

$\checkmark$ Integração com os outros sistemas da UFPB - O ORTODOCS não disponibiliza esse recurso e isso tem como consequência o isolamento, por sua vez o SIGAA se conecta com todos os sistemas integrantes;

$\checkmark$ Emissão de relatórios - Apesar da proposta do ORTODOCS prever a emissão de relatórios, a execução desse recurso é extremamente limitada, deixando a UFPB refém das prioridades dos analistas da Potiron. O SIGAA disponibiliza diversos tipos de relatórios, técnicos e gerenciais em tempo real, que são de extrema importância nas tomadas de decisão;

$\checkmark$ Suporte residente $-\mathrm{O}$ atual sistema de informação da $\mathrm{BC}$, não disponibiliza suporte técnico residente, as demandas são informadas por email e enviadas ao laboratório que tem sede em Campinas - SP, com a assinatura do termo de 
cooperação da UFRN com a UFPB, todo o suporte as demandas operacionais de todos os sistemas SIG, serão atendidas in loco pela equipe do NTI;

$\checkmark$ Custo com renovação - Como o ORTODOCS faz parte de um contrato celebrado entre UFPB e Potiron, tem um custo operacional anual para a instituição. Por outro lado o termo de cooperação que prevê a implantação e transferência de tecnologia dos sistemas SIPAC, SIGAA e SIGRH teve um custo fixo e após o término da cooperação técnica a UFPB terá autonomia na manutenção e desenvolvimento desses sistemas;

$\checkmark$ Atualização de versões com mudanças sugeridas - Partindo do pressuposto que um sistema nunca está completo, surge a necessidade de ajustes periódicos no sentido de aprimorar e melhorar a capacidade do sistema. O SIGAA permitirá essas mudanças com maior flexibilidade que o então sistema ORTODOCS.

Na tabela abaixo identificam-se os pontos estratégicos que não só viabilizam como ratificam a necessidade de mudança, trazendo a esperança de uma integração maior entre os sistemas da UFPB:

Pontos estratégicos que ratificam a mudança de sistema de informação

\begin{tabular}{|l|c|c|}
\cline { 2 - 3 } \multicolumn{1}{c|}{} & ORTODOCS & SIGAA \\
\hline INTEGRAÇÃO COM OUTROS SISTEMAS DA UFPB & NÃO & SIM \\
\hline EMISSÃO DE RELATÓRIOS & NÃO & SIM \\
\hline SUPORTE RESIDENTE & NÃO & SIM \\
\hline CUSTO COM RENOVAÇÃO ANUAL & SIM & NÃO \\
\hline $\begin{array}{l}\text { ATUALIZAÇÃO DE VERSÕES COM MUDANÇAS } \\
\text { SUGERIDAS }\end{array}$ & NÃO & SIM \\
\hline
\end{tabular}

Esses poucos pontos indicados na tabela acima não são os únicos, mas os principais, ratificando que esse enfoque sai da generalidade dos sistemas SIG, para a especificidade do módulo Biblioteca que é o mote desse artigo. O SISTEMOTECA nessa nova formatação terá a possibilidade real de aproximar os segmentos institucionais, mudando dessa forma os atuais procedimentos que só fortalecem o isolamento e falta de unidade. Isso só será possível com o comprometimento dos sujeitos envolvidos. Não há dúvidas quanto à operacionalidade do sistema, que se resume em uma única palavra: INTEGRAÇÂO, das informações, dos 
processos, das tomadas de decisão e das pessoas envolvidas. Como ferramenta de gestão a possibilidade de sucesso existe, mas só se pode validar após o término da efetiva implementação de todos os sistemas.

\section{IMPLANTAÇÃO DO SIGAA (MÓDULO BIBLIOTECA) NA BIBLIOTECA SETORIAL DO CENTRO DE CIÊNCIAS DA SAÚDE}

O Centro de Ciências da Saúde está localizado dentro do Campus I da UFPB e contempla os seguintes cursos: Licenciatura em Educação Física, Terapia Ocupacional, Enfermagem (Bacharelado e Licenciatura), Farmácia, Fisioterapia, Nutrição, Odontologia e Fonoaudiologia, como os outros Centros, possui Biblioteca Setorial. A Biblioteca Setorial do CCS/UFPB surgiu a partir da Biblioteca da pós-graduação em Odontologia do referido Centro. Criada em 1994, funcionou, inicialmente, como Centro de Apoio à Pesquisa da Subrede Nacional de Informação na área de Ciências da Saúde Oral, uma cooperação técnica junto com o Serviço de Documentação Odontológica da Faculdade de Odontologia da USP (SDO/FOUSP), conjuntamente com a BIREME para o estabelecimento do Centro de Apoio à Pesquisa da "Rede Brasileira de Informação em Ciências da Saúde para a área de Odontologia". Atua como biblioteca especializada em Odontologia.

Dentro do Sistema de Bibliotecas a escolha do Centro de Ensino e da Biblioteca como piloto da implantação do módulo, teve como parâmetro a formação do acervo e a disponibilização de serviços informacionais com a mesma estrutura que os oferecidos pela BC. Outro fator relevante na escolha da BS foi o quantitativo de itens que permitiria a migração dos dados do Sistema anterior com maior rapidez e segurança.

O processo e cronograma de implantação dos módulos do SIGAA, foram definidos pelo Núcleo de Tecnologia da Informação - NTI, que a priori estabeleceu o início das

atividades referentes ao módulo Biblioteca para agosto de 2012 e após diálogo com exposição de motivos do SISTEMOTECA, a execução foi antecipada para 07 de fevereiro de 2012. As primeiras reuniões tiveram as finalidades de traçar estratégias para minimizar as dificuldades advindas com a mudança estrutural do sistema e proceder à escolha da Biblioteca Piloto para a efetiva implantação. No dia 10 de abril de 2012 a migração dos dados do ORTODOCS para o SIGAA na Biblioteca Setorial do CCS foi iniciada, o fluxo normal da \begin{tabular}{l|l|l|l|l|l|l|} 
(c) Rev. digit. bibliotecon. cienc. inf. & Campinas, SP & v.11 & n.2 & p.159-175 & maio/ago. 2013 & ISSN 1678-765X \\
\hline
\end{tabular} 
implantação por conta do período de greve foi suspenso sendo retomado no início do mês de setembro, com a ativação da plataforma de testes. No período de 19 a 23 de novembro teve início o treinamento dos bibliotecários do SISTEMOTECA para a utilização do SIGAA.

Durante todo o processo de implantação e treinamentos houve situações que atrasaram o desenvolvimento das atividades, tais como: limitação quantitativa de recursos humanos e período longo de greve. Contudo as atividades do SIGAA na Biblioteca Setorial do CCS foram iniciadas em 10 de dezembro de 2012. A partir dessa data os servidores lotados na BS do CCS estavam capacitados a utilizarem todas as funcionalidades do sistema e o atendimento aos usuários enfim teve seu início, ressaltando que as primeiras reuniões para o planejamento da implantação do sistema no seu módulo Biblioteca, foram realizadas em meados de fevereiro, concluindo-se assim que o processo teve evolução lenta e insatisfatória com reflexos diretos no cronograma de execução.

Esse contexto dificultou a observação dos impactos gerados na estrutura organizacional resultado da implantação do SIGAA em seu módulo Biblioteca e consequentes resultados na sua avaliação como ferramenta de retomada de credibilidade da comunidade acadêmica com a efetivação da gestão do Sistema de Bibliotecas da Universidade Federal da Paraíba.

\section{CONSIDERAÇÕES FINAIS}

Percebe-se que as bibliotecas dos campi inclusive a central, não se encontram ainda com a credibilidade necessária, e isso se verifica nas questões prioritárias dos centros em que elas se encontram. É preciso que se compreenda que uma unidade de informação apesar de ter como matéria-prima os materiais informacionais, elas necessitam do apoio da gestão do centro no sentido de prover condições para que os serviços oferecidos pelas bibliotecas alcancem um nível de satisfação maior dos usuários, e isso não diz respeito apenas aos livros, mas aos equipamentos, a estrutura física, a qualificação dos servidores, a participação dos coordenadores das bibliotecas nas reuniões dos centros. Esse panorama institucional preocupante é na verdade fruto de uma cultura organizacional arraigada e de forte resistência às mudanças.

A transição do atual sistema (ORTODOCS) para o futuro sistema (SIGAA), futuro no módulo Biblioteca, porem já presente na UFPB em alguns outros módulos representa uma \begin{tabular}{|l|l|l|l|l|l|l}
\hline (C) Rev. digit. bibliotecon. cienc. inf. & Campinas, SP & v.11 & n.2 & p.159-175 & maio/ago. 2013 & ISSN 1678-765X \\
\hline
\end{tabular} 
mudança de postura e paradigma de gestão, ousada, mas bem vinda que com certeza será um marco divisório, pois a integração informacional proporcionada servirá não só à transparência, rapidez, segurança, integridade dos processos, mas, sobretudo nas tomadas de decisão com maior segurança e confiabilidade. Vale ressaltar que os detalhes técnicos e características específicas do SIGAA não foram abordados neste trabalho por fazerem parte de um estudo maior a ser desenvolvido posteriormente.

Os resultados observados até o período relatado, não permite uma avaliação ou comprovação da efetividade do novo Sistema de Informação Gerencial o SIGAA, como instrumento de mudança de paradigmas e cultura organizacional na instituição, tendo em vista que o progresso na execução da implantação, treinamento de pessoal através da plataforma de testes e efetivação do módulo em seu perfil de produção, está sendo demasiadamente lento. Esses fatores e acontecimentos não corroboram para uma análise mais fidedigna da eficácia do SIGAA como ferramenta de gestão acadêmica aplicada na instituição, que sirva de parâmetro para justificar o investimento feito na transferência de tecnologia.

O módulo entrou em atividade para os usuários da BS do CCS na primeira quinzena do mês de dezembro de 2012 e permanece em observação para posterior avaliação do custobenefício da transferência de tecnologia promovida pela UFPB, com a finalidade de integrar não só as informações geradas pelos sistemas isolados existentes na instituição mais de aproximar os segmentos através da interação direta entre os gestores e os usuários do SIGAA.

O módulo "Biblioteca", representa uma ferramenta de muitas possibilidades para retomar a credibilidade do SISTEMOTECA, junto aos outros segmentos que fazem parte da academia e assim minimizar esse isolamento ora identificado, ressaltando a necessidade da unidade e não da fragmentação. A Biblioteca Universitária dentro do contexto organizacional nas Instituições Federais de Ensino - IFES tem posicionamento estratégico por estar

relacionada direta ou indiretamente com o tripé: ensino pesquisa e extensão, essa prerrogativa do ponto de vista da Gestão Pública pode ser potencializada com a utilização de um sistema de gestão que integra toda informação gerada e permite aos gestores maior índice de acertos nas decisões tomadas e nas ações executadas. O SIGAA possui essas características e a inclusão da UFPB no rol das IFES que utilizam essa tecnologia demonstra a preocupação institucional em melhorar a qualidade da prestação de serviços aos seus usuários finais. 


\section{REFERÊNCIAS}

AGOSTINHO, M. C. E. Administração complexa: revendo as bases científicas da administração. RAE-eletrônica, v. 2, n. 1, p. 1-18, jan.-jun. 2003.

ASTLEY, W. G.; VAN DE VEM. Debates e perspectivas centrais na teoria das organizações. Revista de administração de Empresa, v. 45, n. 2, 2005.

BERNARDES, C. Teoria geral da administração: a análise integrada das organizações. São Paulo: Pioneira, 1993.

BERNARDES, J. F.; ABREU, FRANCA, A.F.D.; A contribuição dos sistemas de informações na gestão universitária. Disponível em:

<www.inpeau.ufsc.br/.../José\%20Francisco\%20Bernardes\%20-> Acesso em: 10. Jan. 2012.

CUNHA, M. B; A biblioteca universitária na encruzilhada.DataGramaZero-Revista de Ciência da Informação, v.11, n. 6, dez/10.

DAVENPORT, T. H; . Ecologia da Informação: por que só a tecnologia não basta para o sucesso na era da informação. 5. ed. Tradução Bernadete Siqueira Abrão. São Paulo: Futura, 1998. 316 p. Tradução de: Information ecology.

DIAS, M. M.K.; et al. Capacitação do bibliotecário como mediador do aprendizado no uso de fontes de informação. Revista Digital de Biblioteconomia e Ciência da Informação, Campinas, v. 2, n. 1, p.1 -16, jul./dez. 2004. Disponível em:

<http://server01.bc.unicamp.br/seer/ojs/viewarticle.php?id=22\&layout=abstract $>$. Acesso em: 21. jan. 2012.

DUTRA, A. K. F.; OHIRA, Maria Lourdes Blatt. Informatização e automação de bibliotecas: análise das comunicações apresentadas nos seminários nacionais de bibliotecas universitárias (2000, 2002 e 2004). Inf. Inf., Londrina, v.9, n. 1/2, jan./dez. 2004.

FERREIRA, R. S.; A sociedade da informação no Brasil: um ensaio sobre os desafios do Estado. Ci. Inf., Brasília, v. 32, n. 1, p. 36-41, jan./abr. 2003. 
FINGER, A.P.; Liderança e administração na universidade. Florianópolis: UFSC/CPGA, 1986.

FLEURY, M. T. L. Aprendendo a mudar - aprendendo a aprender. Revista de Administração da Universidade de São Paulo, v. 30, n. 3, p. 5-11, 1995.

GALARÇA, S.R.L.; Jornalismo online na sociedade da informação. Dissertação (Mestrado em Ciência da informação) - Universidade Federal do Rio Grande do Sul, Rio Grande do Sul, 2012. Disponível em: www.bibliotecadigital.ufrgs.br. Acesso em: 18 mar. 2012.

GARVIN, D.; Building a learning organization. Disponível em: < http://hbr.org/1993/07/building-a-learning-organization/ar/1 >. acesso em 12. fev. 2012.

GOLD, J. A empresa que aprende baseada no conhecimento. In: CLARKE, T.; MONKHOUSE, E. Repensando a empresa. São Paulo: Pioneira, 1995.

JAPIASSÚ, H.; MARCONDES, Danilo. Dicionário básico de filosofia. 3. ed. Rio de Janeiro: Jorge Zahar, 2001. 296 p.

LAKATOS, E. M.; MARCONI, Marina de Andrade. Metodologia do trabalho científico. 4. ed. São Paulo: Atlas, 1992.

MOIGNE, J. L. A inteligência da complexidade. 2. ed. São Paulo: Petrópolis, 2000, p. 198213.

MORESI, E. A. D.; Delineando o valor do sistema de informação de uma organização. Ci. Inf. , Brasília, v. 29, n. 1, p. 14-24, jan./abr. 2000.

NUNES, P.;Conceito de Enterprise Resource Planning (ERP). 2007. Disponível em: http://www.knoow.net/ciencinformtelec/informatica/enterpresplann.htm

OLIVEIRA, D. de P. R. de.; Sistemas de informações gerenciais: estratégias, táticas, operacionais. 8. ed. São Paulo: Atlas, 2005.

OLIVEIRA, D. de P. R. de.;. Sistemas de Informações Gerenciais:

Estratégicas Táticas Operacionais. 12a Ed. São Paulo: Editora Atlas, 2008, 299 páginas.

PESTANA, M. C.; PIRES, Pedra Margarete de Siqueira Guidil; FUNARO, Vânia Martins Bueno de Oliveira et al. Desafios da sociedade do conhecimento e gestão de pessoas em sistemas de informação. Ci. Inf., Brasília, v. 32, n. 2, p. 77-84, mai./ago. 2003.

RAMOS, M.C. L. A utopia dos bits: impacto das tecnologias de informação na interação bibliotecário/usuário (de graduação) da biblioteca universitária da Universidade Federal de 
Santa Catarina. 2003. 176 f. Dissertação (Mestrado em Engenharia de Produção) Universidade Federal de Santa Catarina, Florianópolis, 2003.

SILVA, A. B.; REBELO, L. M. B. A emergência do pensamento complexo nas organizações. Revista de Administração Pública, Rio de Janeiro, v. 37, n. 4, p. 777-96, jul. -ago. 2003. SILVA, Sergio Luis da. Gestão do conhecimento: uma revisão crítica orientada pela abordagem da criação do conhecimento. Ci. Inf., Brasília, v. 33, n. 2, p. 143-141, mai./ago. 2004.

STAIR, R.M. e REYNOLDS G. W.; Princípios de Sistemas de Informações: Uma abordagem Gerencial. $4^{\circ}$ ed. São Paulo: LTC, 2002.

WEBSTER, F. 2002. Information and postmodernism. Em: WEBSTER, F. Theories of the information society. 2 ed. New York; London: Routledge-USA, 2002, p. 163-192.

\section{Como citar este artigo:}

VIEIRA, Maria das Graças; MACHADO, Fábio Firmino. Sistema Integrado de Gestão de Atividades Acadêmicas SIGAA - Módulo Biblioteca: uma oportunidade de retomar a credibilidade da comunidade acadêmica com a efetivação da gestão do Sistema de Bibliotecas da Universidade Federal da Paraíba. Rev. digit. bibliotecon. cienc. inf., Campinas, SP, v. 11, n. 2, p.159-175, maio/ago. 2013. ISSN 1678765X. Disponível em: <http://www.sbu.unicamp.br/seer/ojs/index.php/rbci> 